\title{
Big Data Analytics and Natural Data Design for Enterprise Management
}

\author{
${ }^{1}$ Zlatan Stojkovic \\ ${ }^{1}$ School of Electrical Engineering, University of Belgrade, Address: 1 Studentski trg, 1000 Belgrade \\ ${ }^{1}$ stojkovic554@hotmail.com
}

\author{
Article Info \\ Journal of Computing and Natural Science (http://anapub.co.ke/journals/jens/jens.html) \\ Doi: https://doi.org/10.53759/181X/JCNS202101014 \\ Received 02 February 2021; Revised form 15 March 2021; Accepted 22 April 2021. \\ Available online 05 July 2021.

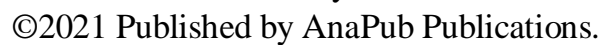

\begin{abstract}
The advancement of Internet of Things (IoT), economic globalization, consumer satisfaction and competitive advancements have stimulated companies to transform significantly. In that regard, competitive rivalry among various firms is being replaced by the existing rivalry among businesses and their various enterprises. In the present competitive environments, enterprise experts are focusing on dealing with Big Data (BD) to reach agile, effective, efficient and integrated enterprises. Therefore, explosive development in volume and several data types in the business have presented the need to establish technological advancements that can quickly and intelligently assess large sets of data. The concept of Data Analytics (DA) is one of the most effective remedies that can assist firms to overcome challenges. DA provides an instrument for retrieving insightful data and patterns in massive volumes of information. In that case, this study explores the usage of DA.
\end{abstract}

Keywords - Big Data (BD), Data Analytics (DA), Internet of Things (IoT)

\section{INTRODUCTION}

Presently, information is advancing rapidly and is projected to reach massive amounts in the upcoming years. The educated population and experts concur that the surge in information creates modern opportunities, and various firms have tried to establish and develop its Data Analytics (DA) capacities, which effectively reveal and reach deep and higher understanding based on the values of Big Data (BD). BD is featured as gigantic or more multifaceted collections of information that typically integrate an extension of exabytes. It normally outstrips the contemporary structures with limited capacities in visualizing, deciphering, overseeing, handling and storing. The study of massive sets of data is persistently extended and advanced, and most of the features of $\mathrm{BD}$ are currently in the $5 \mathrm{~V}$ concept integrating value, volume, velocity, veracity/verification, and variety. DA has been recommended DA as one of the most fundamental factors that influence the performance of business. By progressing DA, firms might effectively comprehend the needs of their consumers, provide more suitable services meant to satisfy the needs, enhance sales, incomes and access newer markets.

Several study studies have shown that BD can be used in a variety of industries, including financial services, marketing, banking, insurance, logistics, and manufacturing, to name a few. The present book chapter, on the other hand, highlights the benefits of $\mathrm{BD}$ usages in terms of establishing novel insights and formulating novel values in approaches that have affected the firms' relations. In that regard, they initially defined DA's major concepts and its major application in foresight. In this paper, the statistical analysis and replication as well as optimization were discussed in detail. The paper also includes a study on the use of DA in DA fields. It then provides a brief overview of how DA can be applied to several types of enterprises, before concluding with a few managerial implications and making suggestions for future study.

First, we need to know what DA is in order to fully appreciate its impact and potential usages. Big Data (BD) can be defined as a large quantity of information. BD is utilized in describing larger sets of data, which are massive and cannot be memorized. This data is easily obtained, stored, transferred, aggregated, and analyzed. The possibilities are limitless! Due to the increasing amount of information available, it has become necessary to update the tools used to analyze it. In order to evaluate it using modern technologies, this information do not need to be laid out neatly in columns and rows like traditional information. BD appears in a wide variety of information types. They use a wide variety of information from a wide variety of sources. It doesn't matter if they're structured or semi-structured. According to another classification, $\mathrm{BD}$ includes numerical information, image information, voice, text, and discourse. A Radio-Frequency Identification Structure (RFID), Point of Sale (POS), Global Positioning Structure (GPS), consumer blogs, Instagram posts, Twitter feeds, Facebook posts and call centers are all examples.

With today's advanced analytical technologies, we're able to extract knowledge from virtually any type of information. Math and statistics are used to analyze large amounts of information. Statistics and mathematics are used to analyze large information sets in DA. When it comes to BD, there is no substitute for analytics. Over the course of several years, the authors have gathered a large amount of data. A collection of statistical and mathematical tools is all 
that analytics is without business intelligence. Companies can use these vast amounts of information to gather intelligence.

A huge amount of computing power is now available at a much lower cost than ever before, which makes it possible for this to occur. When BD and analytics are combined, several tools emerge that assist decision makers gain useful insights and transform information into Business Intelligence (BI). This paper has been organized as follows: Section presents a discussion of business analytics. Section III focuses on the usage areas of DA. Finally, section IV concludes the paper and provides future study directions.

\section{BUSINESS ANALYTICS}

There are many organizations that make up the venture, from manufacturers to production companies, distributors, retail chains, clients, and end customers. Although the enterprise consists of material and artefact flows, it also includes information and financial flows. Using DA techniques, business analytics aims to uncover hidden knowledge from the enterprise. There are three types of analytics that can be used to classify this type of analysis: descriptive, predictive and prescriptive. Planning and implementing decisions that reduce costs of sourcing, transportation, storage, stocking up and disposal have a significant impact on the quality line. As a result of this, DA methodologies used to solve enterprise problems have a positive and significant impact on enterprise performance.

J. Walters and J. Kleijnen in [1] have been using operational and statistical research methodologies to mitigate demand and supply balancing issues for a long time now, and they still do. Analytical techniques have evolved in recent years and are now available to both managers and scholars. Furthermore, scholars investigate how the interactions between descriptive, prescriptive, and predictive modeling can be used to take actions as well as make decisions. Information-supported decision making is gaining attention among academics and researchers because of the numerous benefits that can be realized by using it in SCM. Due to this, the number of research journals regarding this theme has exploded over the past few decades. When it comes to SCM, the importance of using BD Analytical Techniques cannot be overstated. As of 2010, there are a large number of articles on SCM that highlight the use of DA and their significant achievements.

P. Mabry, X. Yan, V. Pentchev, R. Van Rennes, S. McGavin and J. Wittenberg in [2] conducted a bibliographic analysis of BD from 2010 to 2016 to identify influential and prominent scholars as well as the most cited articles. DA has seen a significant increase in the number of articles. In order to study the application of DAs in SCM, scholars carried out a literature review. As a result of the study, predictive and prescriptive techniques are more common in DA than descriptive techniques. Two dimensions of sustainability were examined using BD and prescriptive analytics: social and ecological aspects. Structured equation modeling using partial least squares analyzed the data provided by 205 manufacturing firms.

It was concluded that BD had a significant and positive effect on sustainability's socio-ecological components. On the basis of twenty eight journal articles, the scholars carried out a comprehensive literature review in order to determine the impact of using DA methodologies in humanistic SCM. On the basis of 28 journal articles, scholars examined the use of BD in humanitarian SCM. Several important future research directions were also recommended based on key organization theories such as computational effort and transaction cost economy, and resource administration and resource-based views, and social media network theories and stakeholder theories, among other things.

When it comes to green SCM, V. Gudipati and E. Cha in [3] proposed an optimization framework with multiple objectives that makes use of DA. In order to optimize the inherent threats linked to dangerous materials, material cost and emission of carbon; they looked at three several scenarios. In order to collect and manage the information, they used a BD approach to do so. When it comes to ecological performance evaluation, Song et al. looked at the problems and challenges posed by BD, and summarized recent developments in ecological administration based on BD technologies. The following concerns are addressed in descriptive analysis:

Describe what has occurred, what is currently occurring, and why. On-line analytical processing (OLAP) structures such as GPS RFID and transaction bar-code are being used to develop possible trends and challenges. Statistics that describe the raw information of past events are called descriptive statistics. When it comes to historical events, it describes them, analyzes them, and turns them into something humans can interpret and understand. Companies can learn from the past by using descriptive analytics, which allows them to identify relationships between variables and how they can affect the outcome of the future. In addition to displaying average money and inventory levels, it can also be used to show changes in annual sales. Financial, sales, operations, and manufacturing reports in an organization can also benefit from descriptive analytics.

In predictive modeling, previous information trends are analyzed utilizing mathematical, programming, and computational strategies to address the topic of what will happen tomorrow or likely to occur. With these strategies, you can discover the causes of phenomena and events, effectively forecast the future, or integrate the data that does not yet exist in the first place. Statistical strategies can't be used to forecast with full conviction. Purchase patterns, consumer characteristics and purchasing habits are predicted using predictive analytics to identify and predict future sales trends. These methodologies are often used to anticipate client needs, inventory information, and operational processes.

When it comes to prescriptive analytics, the focus is on what should occur as well as how to impact it. As a result of descriptive and predictive analytics, replication, applied mathematics or multi-approach decision-making methodologies, predictive analysis helps guide possible solutions. In practice, prescriptive analytics is difficult to 
implement, and most companies are still unable to do so. When predictive analytic methodologies are used correctly, it is possible to make appropriate and efficient judgements. DA has been used by a number of large businesses to obtain insights and inventory. Prescriptive analytics allows companies to address a number of critical scenarios, including the following:

- For each end-user, what kind of offer should be made to him?

- When it comes to retail, what should be the shipping strategy for each setting?

- When and what artefact should be launched?

Decision-making in the enterprise is aided by techniques such as analysis of information, replication, and optimization.

\section{Analysis of the Information}

Statisticians distinguish between descriptive and inferential analysis. In order to evaluate or reinterpret a concept, it utilizes flow charts, bar charts, or experimentally analyzed data. A statistical method that relies on past data is used to determine a phenomenon's characteristics and anticipate its behavior. There are differences between descriptive and inferential assessments, according to J. YENSEN in [4]. In order to get the most out of both qualitative and quantitative strategies, they should be used simultaneously. Distribution, stock management and risk management are just a few examples of how statistics are used to deal with uncertainty. As a part of enterprise monitoring, quantitative multivariate methodologies are also used to effectively monitor the process of materials and reduce the risk of unplanned situations occurring.

When it comes to analyzing BD due to its sheer volume as well as its variety and veracity, the enterprise requires robust and easy-to-use techniques. Due to various massive absorption of information, homogeneity, conventional mathematical frameworks are no longer effective. Because of this, constructing and applying effective research method is absolutely critical, and recent attention has been given to this problem. Examples of parallel statistical algorithms are used in study to perform sophisticated statistical analyses of BD. To prototype and compare concentrations and BD allocation squares, this algorithm that uses various methodology like Mann-Whitney U testing, validation, and regression.

\section{Replication}

Simulators are needed by manufacturers to maximize the artefact development, inspire innovation, speed up the time to market, reduce the manufacturing cost, and discover opportunities in their artefacts and processes. Artefact design and manufacturing replications have been shown to have a wide range of benefits at every stage of the process, including the ability to produce more innovative artefacts with greater efficiency and customer satisfaction. During the development of new dishwashing fluids, Proctor \& Gamble employs DA as well as framework construction to anticipate how water will excite definite fragrance particles, allowing them to release the right scents when needed. Simulator and modeling methodologies should be used for developing large information usages like replicationdriven artefact development.

Nowadays, it is a challenge to produce innovative artefacts with the help of simulators. As a result, manufacturers are constantly striving to improve their operational efficiencies, reduce costs, meet deadlines, and anticipate customer preferences. It is possible to run "what-if" analyses under several structure configurations and complexity levels using modeling and replication. To analyze the vast amount of information collected from the surrounding and shop floor environment of a smart manufacturing structure, D. Fournii in [5] developed a replication framework. As a result of this framework, the decision-making process in this manufacturing structure improved. For instance, considered as a predictive instrument, replications are fundamental for manufacturers to effectively forecast the requirements of machines and equipment according on the projections of customers and the learning habits based on historical sets of data e.g. performance of delivery, throughput and cycle time.

Analysts have outlined various examples of where enterprise replications can be applied: result forecast, tests of the inventory policies, evaluating the capacity of manufacturing, determining the usage of assets and validation of results for optimization. Business analytics provide a novel approach for replication issues with a significant amount of dataset. Presently, there is software used for replication, which permit the evaluation of structure performance before its formation. Firm dynamics are one of the incredibly used software usages, which practitioners and scholars utilize when simulating the SCM problems.

\section{Optimization}

The optimization strategy is a critical instrument for the business analytics. The strategies through the extraction of information insights and knowledge of $\mathrm{BD}$ produced by more multifaceted structures, which provide various constraints and factors e.g. route and capacity can evaluate various objectives e.g. cost reduction and order fulfilment. Lastly, utilizing the optimization methodologies in the enterprise alongside the multi-user collaboration, scenario administration and performance trackers allow firms to accomplish their various objectives. The usage of the optimization methodologies are capable of supporting the enterprise planning and also enhances the accuracy of scheduling but presents a larger SCM issues and optimization issues.

D. Yu and L. Deng in [6] have utilized several signal processing and information learning methodologies to analytic optimizations, dictionary training, component evaluation, subspace clustering and detailed sampling. With reference to the SCOR enterprise framework, the authors evaluated the available chances of utilizing DA in SCM. DA is a fundamental tool when applied in various operational, strategic and tactical levels within the organization's 
enterprise. For instance, within the strategic level, the business analytics is utilized for sourcing, network design, and artefact designing; within the operational and tactical levels; business analytics are applied in inventories, logistics, demand planning, and procurement.

\section{APPLICATION}

Within the manufacturing department, BD is produced by both the external networks and internal networks, which incorporate the sensor networks or the instrumentation within the manufacturing level. The usage of BD to sophisticated integration and analysis of databases can aid in the improvement of distribution efficiency and enhance the processing of dales and progressive monitoring of devices and business processes. Manufacturing firms have to utilize BD and the various analytics approaches to develop their processing department. Predictive maintainability of instruments is a segment in this department to enhance business development. Because of the massive numbers of vendors, and the several selections and evaluation indicators, the process of choosing the optimal and right vendors for the enterprise is a challenging task. The usage of cloud computing in the selection of the right vendors is fundamental. With the novel structures, exposure and access to BD is significantly intuitive and consumer-centred with the power of API and the incorporation of modern BD usage and DA packages. DA can be applied in several fields:

\section{Distributer Relationship}

The administration of the distributer relationship integrates the establishment of a discipline in strategic administration and planning of various interactions with firms' suppliers to minimize the risks of failure and maximize the interaction values. Enhancing a closer connection with the major distributers and developing the association with them are a fundamental factor in the creation and discovery of novel value and minimizing the risks of failure in the distributer relationship administration. Distributer relationship administration and strategic resources are the success factors of firms that concentrate of relationship collaboration and administration.

It is possible to obtain more precise insight into business spending habits by using DA techniques, which helps in proper management of the distributor relationship. The BD can, for example, provide more reliable information about return on investment (ROI) for different investments and a detailed analysis of the possible vendors. R. Ohta in [7] used the Analytical Hierarchy Process (AHP) and the Fuzzy Synthetic Evaluation (FSE) to the selection and assessment of distributers, provided the capability of BD processing as one of the assessed elements. The main purpose for this was to choose the supply partners, which are capable of adapting to upcoming issues from BD.

\section{Enterprise Networking Designs}

The designing of the enterprise is a fundamental decision that incorporates the various decisions concerning the selection of key partners within the enterprise and defining the organizational protocols and programs meant to accomplish long-term strategic objectives. Enterprise networking design project integrates the determination of the enterprise natural configurations, which influence several organizational units or the critical segments within the firm. In the design of enterprise networks, it is fundamental to determine the enterprise efficacy and client satisfaction. The obligation of enterprise designing is to formulate the members' networks, which are capable to accomplish the longlasting strategic targets of the firm. During the designing of enterprises, some of the steps are considered:

- Defining the long-lasting strategic targets;

- Defining the entire scope of projects;

- Determining the type of analyses that have to be done;

- The instruments, which will be utilized have to be determined; and

- $\quad$ Task completion, the most effective design.

Choosing the optimum enterprise designing and effective scheduling, the firm is capable of accomplishing a fundamental competitive advantage. S. Salahi and S. Bahramara in [8] have projected a mixed-integer non-linear framework that is capable of locating the center of distribution, using BD in this framework, and generating massive sets of data in a random manner for the various operations in the warehouse, transportation and customer demand. The scholars assume that the action sets of data have been evaluation based on the usage of smart tools. As a result of their findings, BD appears to be a powerful tool for multifaceted distribution network designs, as it can provide critical data about penalities costs and service dimensions. The study evaluates the usage of BD analytics in the design interventions e.g. education, disaster relief and healthcare in the enterprise. Since the humanitarian information have the features of speed, accuracy, high diversity, and high volume, BD analytics can be utilized in the humanitarian enterprises.

\section{Artefact Designing and Artefact Improvement}

Processors of resilient artefacts must ensure that the artefacts meet the preferences of customers. As customers' preferences and expectations change over the course of a product's lifespan, designers need instruments that can measure and predict these changes. The lack of enough information on clients' expectations and preferences is a fundamental concern in the process of artefact designing. In case designers progressively track the behaviours of clients and access updated information regarding the behaviour of clients, they can effectively design artefacts, which meet the expectations and preferences of customers. Progressive tracking of clients' behaviours, manufacturing processes and artefact design generate massive sets of data that are considered as BD 
It is possible to reduce uncertainty by managing, collecting, and implementing novel analytical techniques to gain insights and sensitive material, and then implementing them to the decision-making method. Engineering designing is illustrated as a procedure of changing the needs of customers into designing specifications. Information science is defined as a procedure of changing the observed globe realistic information into comprehensible information for the purpose of making informed decisions. Even though various methodologies are present for artefacts' designs, all the approaches are common in the perspective of information sciences. Massive sets of data are going to have a significant implication in various industries, and artefact designing is no exception. This is important because engineers will be designing sensors and communication initiatives into their artifacts over the course of time. When it comes to enterprise design, it's important to take into account the specifics of the firm's artifacts and to include all of its partners, as well as its constraints.

Enterprise designing, in reference to artefact design, establishes flexibility and competitive advantage within the enterprise. In recent times, DA methodologies have been utilized for artefact designing and development that amount to the manufacturing of novel artefacts based on the preferences of customers. Incorporating DA into artefact designing enables developers to become increasingly aware of customer expectations and preferences. As a result, artefacts can be manufactured based on consumer choices. Designers can utilize online customers purchasing records and customer behaviours to forecast and comprehend the needs of clients. These designers can identify the features of artefacts and forecast the future trends of artefacts by progressively tracking the behaviours of consumers and informing the clients' needs and opinions.

Its importance in the automotive industry comes from the motors, which provide important client needs and performance information. The ultimate goal of companies that produce consumer durables is to maintain their competitiveness for as long as possible. In the present times, this facilitates the usage of the ideology of "run-time" information-oriented designs. Over the past few decades, the development of DA and the integration of analytics instruments have created novel paths for providing knowledge for artefact development and accomplishing strategic objectives. As a single doctrine, the developers of artefacts can accomplish perpetual development of their services and artefacts with respect to real-life usage, failure and work information. Based on wide-range DA, packages and tools have been put in place to extract artefact-based information, applying DA methodologies and tools in artefact development; however, this is still in its premature phase.

Artefact designers are still facing numerous issues and have to consider wide-range limitation. It is reported that selecting the most fundamental tools for DA and utilizing them in the design projects are non-trivial for artefact designers. Below are some of the other strategies design engineering could transform due to the $\mathrm{BD}$ it allows:

- Sophisticated development of artefacts - How could the means firms formulate artefacts' transition if they would master not only how clients are utilizing them and what characteristics they are have issues with them and the kind of features they are ignoring? This information will be available to firms in the near future. Mechanical engineers are provided with the chance for artefact insights, which were impossible earlier on. Internet of Things (IoT) devices allows artefacts to transmit usage data back to their designers. Consider, for example, a bike fork that can record force measurements, or a cabinet that can transmit internalized temperature records, as examples.

- Empowered engineering - Contemporarily, artefact engineers depend on client visits, advertisers or their guesses for designing competitive artefacts. Nonetheless, BD could effectively artefact wide-range dependable responses, which none of these channels produce. Even though artefacts produce massive amounts of data over the course of their lifespan, the IoT will provide even more information to manufacturing firms. Data from interconnected devices will be used to create consumable information assets. In the future, artefacts will be able to communicate and interact with their designers, giving the designers a greater influence on the artefact's competitiveness.

- Prompt artefact engineering and development - In as much as BD is in the cloud structure, more individuals can safely access information faster (and at an affordable rate) than working within specific platforms or corporate network structures. This might amount to more disciplines and participants associate in the development cycle of artefacts earlier on. New methods for more simultaneous CAD designs and constructions engineering protocols that integrate electrical, structural, and operating systems usage in the advancement of artifacts will be presented by the IT infrastructure of cloud computing [12].

Finalizing the artefact design process, engineers will be faced with a variety of surprises and unwanted shocks as distinct disciplines interlink and access BD throughout the different stages of the design cycle. The actual issue will be focussed on mitigating these hassles and in the development of effective engineered artefacts accessing novel levels in the designing of artefacts [13].

\section{Demand Scheduling}

Various enterprise leaders are careful at enhancing demand manufacturing and forecasting scheduling with massive. Precise demand planning has always been a significant concern in the administration of the SCM. Trace consumer loyalty information, optimal price information and demand signals information can be obtained by DA. In spite of this, firms have a hard time implementing more advanced software and hardware, including algorithm architecture. 
DA permits the identification of novel trends in the market, and determination of the root causes of defects, failures and issues.

DA could forecast clients' needs and preferences by evaluating clients' behaviours that can initiate innovation and creativity in organizational services. R. Lee and S. Brown in [9] presented a framework to forecast electric motor charging demands, which utilized weather information and historical actual-world traffic sets of data. Due to the determination of the charging demands, operators are able to effectively plan generation profiles and schedule operations. There is also a framework for forecasting the demands for air passenger demands that utilize BD for evaluating the air passenger demands. The findings in this study indicate a 5\% forecasting error.

\section{Procurement Administration}

Just like operational and tactical decisions, procurement integrates a collection of action mechanisms and contracting. Logistic firms provided the high volumes of disseminated information produced across multiple structures, geographical parts, and operations necessitate more advanced structures to effectively manage BD and skilled experts who can evaluate these sets of information, and extract useful insights and skillset into them to apply them in decision-making processes and planning. Earlier on, firms faced laborious procedures that tool various weeks to collect structural and internal information from transactions and operations of the firms and their stakeholders. All of the internal, external, structural, and non-structural data generated by automated processes is now accessible to these companies at an exponentially.

Distributors' efficiency and innovation risk management can be effectively managed using business analytics. Business intelligence (BI) tools, both internal and external, have been used to manage and address problems in the organization. For instance, informing the news and social media platforms about the rates of exchange disasters and movements influences the entire enterprise. The usage of this framework to identity the risks in the enterprise enables actual-time risk administration monitoring, emergency planning and decision-making support. S. Mathrani and X. Lai in [10] proposed a BD predictive analytic framework for identifying, evaluating, mitigating and managing the risks in the enterprise.

\section{Customized Manufacturing}

It is possible for manufacturers to discover new information and patterns using DA in order to develop processes, improve the efficiency of enterprises and identify potential variables that affect manufacturing. Due to today's interconnected global environment, manufacturing procedures and enterprise procedures have to be able to evaluate the various elements of each procedure as well as connect them in granular detail to simplify and optimize them. DA allows manufacturers to precisely determine every individual's tasks and activities through accurate and timely information evaluation of every segment of the manufacturing process and evaluate the complete enterprise comprehensively. This capacity allows manufacturers to effectively evaluate the bottlenecks and identify the ineffectively performing elements and processes.

Over the past few decades, centralized manufacturing and manufacturing for business analytics were non-rationale due to constant focus on the ordering of minor teams of consumers, whereas today's DA have facilitated the accurate prediction of consumer tastes and demands for customized engineered artefacts. Z. Azough in [11] has conducted an evaluation of the applied methodologies of DA in the manufacturing sector. They applied RFID-driven BD to support shop floor logistic scheduling and planning. In the process, they implemented the physical internet ideology to applying IoT, DA and wireless technology to formulate RFID-driven smart shop flow environment. DA methodologies have been utilized in forecasting manufacturing and demand levels in manufacturing firms.

Contrary to that, initial addicting manufacturing (also known as three-dimensional printing) was formulated in 1980s. The novel trends and technologies are rapidly emerging, which will transform the protocols of the SCM and designing. Three-dimensional printing is any of the several producers whereby substances are solidified or interlinked under the control of computing to formulate three-dimensional objects. Three-dimensional printing is a technology, which makes it possible to formulate physical objectives from digitalized frameworks. Comprehending the usages and effectives of predictive analytics and BD will be required as additive processing makes contemporary frameworks of manufacturing, supply and demand more absolute in various artefact segments.

\section{CONCLUSION AND FUTURE DIRECTIONS}

Data Analytics (DA) and its use in Supply Chain Administration are examined in depth in this paper (SCM). DA has now become an important practical concern in various segments of the enterprise, as this paper demonstrates. There are multiple scopes for the development of usage of effective analytics methodologies in these segments. From this analysis, it is evident that there are various fundamental usages and techniques in the SCM such as prescriptive, descriptive and predictive). This paper has shown some of the usages of DA in the administration of enterprises as well as noticing some of the fundamental methodologies in the SCM, which are fundamental for organizational objectives.

DA has significant usage across the enterprise, which integrates the demand information within the firms' sales department, manufacturing information, delivery information, retailer information and the distributer information. DA are utilized in several enterprise endeavours such as distribution, logistics, procurement, manufacturing, network designing, inventory, demand scheduling, artefact designing and development and distributer relationship administration. The usage of BD sources and analytics methodology has amounted to numerous developments in the 
enterprise producers. Moreover, DA can effectively support the enhancement and improvement of reliable, sustainable and responsive enterprises. DA can allow the administration and integration of massive amounts of diversified information in more multifaceted global enterprises. From this study, it is evident that most scholars have utilized several methodologies of DA across multiple firms such as banking, manufacturing and healthcare industries. Other firms e.g. service, energy, Internet of Things (IoT) and hospitality industries will also take full advantage of DA methodologies.

Based on the contexts applied and the strategic needs of firms, various methodologies of DA are used. The administration, environment, policies and cultural groups within the firm are fundamental factors in the process of decision-making. Enough resources with analytics capacities have become a major challenge for most of today's enterprises. In that case, future study should focus on study that will help enterprises to establish closer and progressive connections between information professionals and their organizational functions while also applying effective DA methodologies based on the context of their usage in the process of decision-making including the activities meant to determine how $\mathrm{BD}$ can drive results within the enterprise. Therefore, mutual cooperation and coordination between various units of the enterprise should be established, utilize DA methodologies to connect their units and present the capacity to access and share information within the organization.

\section{References}

[1]. J. Walters and J. Kleijnen, "Statistical Techniques in Simulation", Operational Research Quarterly (1970-1977), vol. 27, no. 2, p. 518 , 1976. Available: $10.2307 / 3008825$.

[2]. P. Mabry, X. Yan, V. Pentchev, R. Van Rennes, S. McGavin and J. Wittenberg, "CADRE: A Collaborative, Cloud-Based Solution for Big Bibliographic Data Research in Academic Libraries", Frontiers in Big Data, vol. 3, 2020. Available: 10.3389/fdata.2020.556282.

[3]. V. Gudipati and E. Cha, "Target reliability index optimization framework for multiple building classes based on community-level objectives", Structural Safety, vol. 91, p. 102097, 2021. Available: 10.1016/j.strusafe.2021.102097.

[4]. J. YENSEN, "Complete Report of Descriptive and Inferential Statistics Requested", Nursing Research, vol. 28, no. 2, p. 80, 1979. Available: 10.1097/00006199-197903000-00004

[5]. D. Fournii, "A Self-Contained Framework for Replication with Funded Assets", SSRN Electronic Journal, 2016. Available: $10.2139 /$ ssrn.2755746.

[6]. H. Anandakumar and K. Umamaheswari, "Supervised machine learning techniques in cognitive radio networks during cooperative spectrum handovers," Cluster Computing, vol. 20, no. 2, pp. 1505-1515, Mar. 2017.

[7]. H. Anandakumar and K. Umamaheswari, "A bio-inspired swarm intelligence technique for social aware cognitive radio handovers," Computers \& Electrical Engineering, vol. 71, pp. 925-937, Oct. 2018. doi:10.1016/j.compeleceng.2017.09.016

[8]. D. Yu and L. Deng, "Deep Learning and Its Applications to Signal and Information Processing [Exploratory DSP", IEEE Signal Processing Magazine, vol. 28, no. 1, pp. 145-154, 2011. Available: 10.1109/msp.2010.939038.

[9]. R. Ohta, "Selection Of Industrial Maintenance Strategy: Classical Ahp And Fuzzy Ahp Applications", International Journal of the Analytic Hierarchy Process, vol. 10, no. 2, 2018. Available: 10.13033/ijahp.v10i2.551

[10]. S. Salahi and S. Bahramara, "Modeling Operation Problem of Micro-grids Considering Economical, Technical and Environmental issues as Mixed-Integer Non-Linear Programming", International Journal of Renewable Energy Development, vol. 5, no. 2, pp. 139149, 2016. Available: 10.14710/ijred.5.2.139-149.

[11]. R. Lee and S. Brown, "Evaluating the Role of Behaviour and Social Class in Electric Vehicle Adoption and Charging Demands", SSRN Electronic Journal, 2020. Available: 10.2139/ssrn.3724667.

[12]. S. Mathrani and X. Lai, "Big Data Analytic Framework for Organizational Leverage", Applied Sciences, vol. 11, no. 5, p. $2340,2021$. Available: 10.3390/app11052340.

[13]. Z. Azough, A. Benomar and M. Bellafkih, "Data Analytics Applied to Scholarly Data Moroccan Research Trends", SSRN Electronic Journal, 2018. Available: 10.2139/ssrn.3187031. 